\title{
Composite Fermions with Orbital Magnetization
}

\author{
Steven H. Simon ${ }^{1}$, Ady Stern ${ }^{2}$, and Bertrand I. Halperin ${ }^{3}$ \\ ${ }^{1}$ Department of Physics, Massachusetts Institute of Technology, Cambridge, MA 02139 \\ ${ }^{2}$ Department of Condensed Matter Physics, Weizmann Institute of Sciences, Rehovot 76100, Israel \\ ${ }^{3}$ Department of Physics, Harvard University, Cambridge, MA 02138
}

\begin{abstract}
For quantum Hall systems, in the limit of large magnetic field $B$ (or equivalently small electron band mass $m_{b}$ ), the static response of electrons to a spatially varying magnetic field is largely determined by kinetic energy considerations. This response is not correctly given in existing approximations based on the Fermion Chern-Simons theory of the partially filled Landau level. We remedy this problem by attaching an orbital magnetization to each fermion to separate the current into magnetization and transport contributions, associated with the cyclotron and guiding center motions respectively. This leads to a Chern-Simons Fermi liquid description of the $\nu=\frac{1}{2 m}$ state which correctly predicts the $m_{b}$ dependence of the static and dynamic response in the limit $m_{b} \rightarrow 0$.
\end{abstract}

A useful theoretical tool for studying quantum Hall states is the composite fermions picture, known also as the Chern-Simons fermionic theory [1]. Based on the work of Jain [2], the Chern-Simons fermion picture was introduced by Lopez and Fradkin [3] to study fractional quantized Hall states, and by Halperin, Lee and Read (HLR) [1], as well as Kalmeyer and Zhang [4], to study even denominator filling fractions.

In the composite fermion description, a system of spin polarized electrons in a two dimensional electron gas is transformed exactly into a system of composite, or gaugetransformed, fermions. These composite fermions carry an even number $\tilde{\phi}=2 m$ of 'Chern-Simons' flux quanta and interact with each other both electrostatically and via the Chern-Simons gauge field [1]. At a mean field level the fermions are subject to a uniform effective magnetic field $\Delta B=B-\Phi_{0} n_{e} \tilde{\phi}$ where $\Phi_{0} \equiv h / e$ is the flux quantum, and $n_{e}$ is the average electron density (the speed of light $c=1$ ). Such an approximation maps electrons at filling fraction $\nu=\frac{1}{2 m}$ to fermions in zero magnetic field, where the ground state should be a gapless Fermi liquid-like state. Similarly, this transformation maps [1.3] fractional quantized Hall states at filling fractions $\nu=\frac{p}{2 m p+1}$ to integer quantized Hall states of fermions at $\nu=p$.

The main systematic attempts for going beyond mean field theory have so far involved perturbative treatments of the Chern-Simons and electrostatic interactions. [1.66 8] Attention has focused on one difficulty the divergence of the composite fermion's effective mass at the Fermi surface when the range of electrostatic interaction is shorter than, or equal to, that of the Coulomb interaction. This divergence, due to infra-red gauge field fluctuations, is manifested in the energy gaps of fractional quantized Hall states at $\nu=\frac{p}{2 m p+1}$ (for large $p$ ) but does not affect the electronic linear response at $\nu=\frac{1}{2 m}$ at zero temperature, due to a mutual cancelation with another singular term [7,8]. Consequently [7] the low energy excitations at $\nu=\frac{1}{2 m}$ are best characterized by another, finite, effective mass, denoted by $m^{*}$, which is the effective mass of relevance to the present work. It is this $m^{*}$ which should determine the scale of the fractional Hall gaps for small values of $p$.

In this paper we consider a second source of difficulty in the Chern-Simons theory which appears even when gauge-field fluctuations are not infra-red singular. (For example, there is no predicted infra-red divergences in the effective mass if the electron-electron repulsion falls off more slowly than $1 / r$.) This second difficulty is encountered in the limit of a small band mass $m_{b}$ or equivalently the large $B$ limit. The fact that, in this limit, the electronic ground state and low energy excitations are constrained to the lowest Landau level, leads to certain features of the electronic response to an external static vector potential which are not properly embodied in previous approximation schemes. In this paper we suggest a remedy to that flaw. We argue that while previously used approximations are appropriate for calculating the density-density response function $K_{00}$ (defined below) they are not appropriate for response functions such as the density response to a transverse vector potential $K_{01}$. The approach we present allows us to derive an approximation to the linear response function which yields the correct $m_{b}$ dependence in the limit $m_{b} \rightarrow 0$.

Our approach is based on a separation of the current into a magnetization current which is associated with the cyclotron motion of electrons and a transport current associated with the guiding center motion. This separation is achieved by attaching a magnetization $\mu_{\mathrm{M}}$ to each particle. This magnetization originates from the electrons' orbital motion and is unrelated to the spin (which is assumed to be a frozen degree of freedom). In the limit $m_{b} \rightarrow 0$, the magnetization $\mu_{\mathrm{M}}$ is given by the Bohr magneton $\mu_{b}=e \hbar /\left(2 m_{b}\right)$. Our separation procedure, combined with approximations similar to those made in previous works, results in response functions that correctly describe the $m_{b} \rightarrow 0$ limit.

We will mostly discuss the behavior of the electromagnetic response matrix $K_{\mu \nu}$ which is closely related to the conductivity [1.6]. To define $K$, a weak vector potential $A_{\mu}^{\text {ext }}$ is externally applied to a system at wavevector $\mathbf{q}$ and frequency $\omega$, and consequently, a current $j_{\mu}$ is induced (Here $A_{0}$ is the scalar potential, and $j_{0}$ is the induced density). We write the response function in the 
form $j_{\mu}(q, \omega)=K_{\mu \nu}(q, \omega) A_{\nu}^{\operatorname{ext}}(q, \omega)$ where $\mu$ and $\nu$ take the values $0, x, y$. We will use the convention that the perturbation is applied with $q \| \hat{\mathbf{x}}$ so that the longitudinal current is $j_{x}=(\omega / q) j_{0}$. Using the gauge $\mathbf{A}_{x}=0$, we can then treat $K_{\mu \nu}$ as a $2 \times 2$ matrix with indices taking the values 0 or 1 denoting the time or transverse space components. In this notation the current vector $j_{\mu}$ is $\left(j_{0}, j_{y}\right)$, and the vector potential $A_{\mu}$ is $\left(A_{0}, A_{y}\right)$. In the HLR approach [1], the response matrix $K$ is calculated within the RPA. As reviewed below, an improved 'Modified' RPA (MRPA) was introduced in later work [6].

To understand why we believe the RPA and MRPA miss some important physics, we consider a system at $\nu=\frac{1}{2}$ such that the magnetic field is $B_{1 / 2}=2 n_{e} \Phi_{0}$. We now apply a weak static field $\delta B(\mathbf{r})$ at some small but finite wavevector $q$. At a mean field, RPA, or MRPA level, the transformed fermions see only the additional field $\delta B(\mathbf{r})$, and the energy is the same in regions where $\delta B>0$ as it is in regions where $\delta B<0$. However, the original electrons see the full field $B_{1 / 2}+\delta B(\mathbf{r})$. Since the electrons are in the lowest Landau level they have a kinetic energy $\frac{1}{2} \hbar \omega_{c}(\mathbf{r})=\mu_{b}\left(B_{1 / 2}+\delta B(\mathbf{r})\right)$ which is lower in regions where $\delta B<0$. In the limit of $m_{b} \rightarrow 0$, the electrons are then strongly attracted to regions where $\delta B(\mathbf{r})<0$. This behavior should be reflected in $K_{01}$, but is not accounted for at a mean field or (M)RPA level.

In order to model this attraction to regions of minimal magnetic field, we attach to each particle a fixed magnetization $\mu_{\mathrm{M}}$ which interacts with the magnetic field and creates an energy cost $\mu_{\mathrm{M}} B(\mathbf{r})$ as a function of position. In the limit where the interaction energy goes to zero (or equivalently if $m_{b} \rightarrow 0$ ), $\mu_{\mathrm{M}}$ is given by $\mu_{b}$, so that this energy cost consists of the kinetic energy only and is given by $\mu_{\mathrm{M}} B(\mathbf{r})=\frac{1}{2} \hbar \omega_{c}(\mathbf{r})$. For finite interaction energy the local energy cost is composed of kinetic and interaction contributions. Within our framework, most of that energy should be described as magnetization energy, with the remaining part described as quasiparticle interaction energy. There is, however, some arbitrariness in that separation, and consequently in the definition of $\mu_{\mathrm{M}}$. For the present purpose we need to consider only the leading behavior in $1 / m_{b}$ and we can therefore put $\mu_{\mathrm{M}}=\mu_{b}$. Following the attachment of magnetization, we define an effective scalar potential seen by the magnetized particles as $A_{0}^{\mathrm{eff}}=A_{0}+\mu_{\mathrm{M}} B(\mathbf{r})$ and declare that the particles respond to this effective field.

Another effect that is not accounted for in previous works involves the magnetization current, whose physical interpretation is the following. In a high magnetic field, each particle can be thought of as traveling in a cyclotron orbit. When the density of particles is uniform, the local currents of these orbits cancel and there is no net current in the bulk of the system. However, when there is a density inhomogeneity, these local currents do not quite cancel and a net magnetization current results. Formally, we write $\mathbf{j}_{\text {mag }}=\nabla \times \mathbf{M}$ where $\mathbf{M}$ is the magnetization density which is given, for non- interacting particles in the lowest Landau level, by $\mathbf{M}=$ $\frac{1}{2} \hat{\mathbf{z}} \hbar \omega_{c}(\mathbf{r}) n(\mathbf{r}) / B(\mathbf{r})=\mu_{b} n(\mathbf{r})\left(\right.$ where $n(\mathbf{r})=n_{e}+j_{0}(\mathbf{r})$ is the local density). Again, more generally $\mu_{b}$ might be replaced by $\mu_{\mathrm{M}}$ such that $\mathbf{M}=\mu_{\mathrm{M}} n(\mathbf{r})$. Thus, the magnetization current is $\mathbf{j}_{\text {mag }}=\mu_{\mathrm{M}}(\hat{\mathbf{z}} \times \nabla n(\mathbf{r}))$. By attaching magnetization $\mu_{\mathrm{M}}$ to each particle, which is equivalent to attaching a current loop, we separate out the contribution of the magnetization current to the total current. It is then convenient to define the transport [9] current $\mathbf{j}_{\text {trans }}=\mathbf{j}_{\text {total }}-\mathbf{j}_{\text {mag }}$. The transport current may be interpreted as the current of the bound particle-magnetization composite objects.

When projected to the lowest Landau level, the projected current and density operators satisfy $P \mathbf{j} P=$ $\mu_{b}(\hat{\mathbf{z}} \times \nabla P n P)$, ie, the projected current operator describes only magnetization current [10] (Here, $P$ is the projection operator). Therefore, in the limit $m_{b} \rightarrow 0$, if we apply a static scalar potential $A_{0}^{\text {ext }}(q)$ to the system and we look at the current response in powers of $1 / m_{b}$, we find a transverse magnetization current $\mu_{b} \hat{\mathbf{z}} \times i \mathbf{q} K_{00} A_{0}^{\text {ext }}$ originating from lowest Landau level matrix elements, as well as a current independent of $m_{b}$, which may be described as arising from virtual transitions to other Landau levels. Thus, if $q$ is finite and $m_{b} \rightarrow 0$, we expect $K_{10} / K_{00} \rightarrow i q \mu_{b}$. This result is not contained in previous works based on the Chern-Simons approach.

Before detailing our remedies to the above problems, we review the RPA and MRPA. Both approaches separate the long ranged interactions by writing $K$ as $6,7,10,11$

$$
K^{-1}=\Pi^{-1}+U \quad \text { where } \quad U=\left(\begin{array}{cc}
v(q) & -c(q) \\
c(q) & 0
\end{array}\right) .
$$

Here $v(q)=e^{2} /(\epsilon q)$ is the Coulomb interaction and $c(q)=i \tilde{\phi} \Phi_{0} / q$ is the Chern-Simons interaction. In other words, $\Pi$ is the part of $K$ that is irreducible with respect to both $v(q)$ and $c(q)$. The RPA [1,3] is obtained by setting $\Pi$ equal to $K^{0}$, the response of a noninteracting system of fermions with mass $m_{b}$ in the mean magnetic field $\Delta B$. This RPA approach has the problem that the low energy excitations are on a scale set by the cyclotron energy (ie, by $m_{b}$ ) rather than by the interaction energy. The MRPA [6] repairs this problem by defining an effective mass $m^{*}$ that is set phenomenologically by the interaction scale [1]. Galilean invariance is maintained by adding a Landau interaction term $\mathcal{F}_{1}$ to insure that Kohn's theorem and the $f$-sum rule will be satisfied. To define the MRPA we write

$$
\begin{aligned}
\Pi^{-1} & =\left[\Pi^{*}\right]^{-1}+\mathcal{F}_{1} \\
\mathcal{F}_{1} & =\frac{\left(m^{*}-m_{b}\right)}{n_{e} e^{2}}\left(\begin{array}{cc}
\frac{\omega^{2}}{q^{2}} & 0 \\
0 & -1
\end{array}\right) .
\end{aligned}
$$

The MRPA is then obtained by setting $\Pi^{*}$ equal to the response $K^{0 *}$ of a system of noninteracting fermions of mass $m^{*}$ in the mean magnetic field $\Delta B$. The response function thus calculated (using $\Pi^{*}=K^{0 *}$ and Eqns. 2 
and (1) will be called $K^{\mathrm{MRPA}}$. Comparisons of numerical results of exact diagonalizations to results of $K_{00}$ calculated in the MRPA were quite favorable [6] for the low energy excitations at $\nu=\frac{p}{2 m p+1}$ for small $p$.

As described above, the (M)RPA approach does not account for magnetization effects due to the fact that when we take the mean field solution as a starting point for a perturbation theory for the Chern-Simons fermions, we lose the fact that the original electrons travel in local cyclotron orbits. This piece of physics would presumably be recovered if one could correctly carry out a renormalization procedure which eliminates the high frequency motion at the cyclotron frequency to obtain equations valid on the scale of the Coulomb interaction. In the current work, we recover this physics by attaching magnetization to each particle by hand. This attachment is not an exact transformation, but is rather a way of modeling behavior that is lost when we take the mean field as a starting point. As we will see below, within a Landau-Fermi liquid theory picture, this attachment seems to give the correct quasiparticles for the system.

The attachment of magnetization to the particles does not change the interparticle interactions. However, the magnetized fermions now respond to the effective potential and the motion of these magnetized fermions yields only the transport current response. We thus define a matrix $\tilde{K}$ to be the transport current response of the electrons to the external effective potential. We thus write

$$
\begin{array}{cc}
A_{\text {eff }}= & M^{\dagger} A \\
j_{\text {total }} & =M j_{\text {trans }}
\end{array} \quad M=\left(\begin{array}{cc}
1 & 0 \\
i q \mu_{\mathrm{M}} & 1
\end{array}\right)
$$

and relate $\tilde{K}$ to $K$ by $K=M \tilde{K} M^{\dagger}$. As discussed above, in the limit $m_{b} \rightarrow 0$, we must have $\mu_{\mathrm{M}} \rightarrow \mu_{b}$. In the rest of this paper, however, we will consider $\mu_{\mathrm{M}}=\mu_{b}$.

We now propose an approximation which we call the 'Magnetized Modified RPA' or $\mathrm{M}^{2} \mathrm{RPA}$ where we set $\tilde{K}$ equal to $K^{\mathrm{MRPA}}$. Thus in the $\mathrm{M}^{2} \mathrm{RPA}$ we approximate

$$
K \approx M\left(\left[K^{0 *}\right]^{-1}+\mathcal{F}_{1}+U\right)^{-1} M^{\dagger} .
$$

This equation is a central result of this paper. In the limit $m_{b} \rightarrow 0$, the $\mathrm{M}^{2} \mathrm{RPA}$ correctly describes the static response properties described above. It should be noted that the $\mathrm{M}^{2} \mathrm{RPA}$ predicts $K_{00}=K_{00}^{\mathrm{MRPA}}$ and is therefore equally well supported by exact diagonalizations [6]. Furthermore, we note that the excitation modes of a system are determined by the poles of $\operatorname{det}[K]$, and since $\operatorname{det}[K]=\operatorname{det}[\tilde{K}]$, the MRPA and the $\mathrm{M}^{2} \mathrm{RPA}$ predict the same excitation energies. However, the $\mathrm{M}^{2} \mathrm{RPA}$ calculations of $K_{01}$ and $K_{10}$ differ from $K_{01}^{\mathrm{MRPA}}$ and $K_{10}^{\mathrm{MRPA}}$ by terms of order $q / m_{b}$ and the $\mathrm{M}^{2} \mathrm{RPA}$ value of $K_{11}$ differs from $K_{11}^{\mathrm{MRPA}}$ by terms of order $q / m_{b}$ and by terms of order $\left(q / m_{b}\right)^{2}$. It should be noted that all finite $q$ experimental tests [0] of the Chern-Simons theory to date have measured only $K_{00}$. Similar to MRPA, we expect the $\mathrm{M}^{2} \mathrm{RPA}$, in addition to describing the $\nu=\frac{1}{2 m}$ Fermi liquid states, should properly describe the Jain series of quantized states $\nu=\frac{p}{2 m p+1}$ for small $p$. At large values of $p$, the description should be modified to account for the effects of the singular infra-red gauge fluctuations.

We now turn to discuss how the $\mathrm{M}^{2} \mathrm{RPA}$ fits into the general picture of a Fermi liquid theory of the $\nu=1 /(2 m)$ state. In essence, we show that $\mathrm{M}^{2} \mathrm{RPA}$ amounts to adopting the Fermi liquid picture of Ref. [7] as describing the dynamics of magnetized composite fermion quasiparticles rather than unmagnetized ones.

In Fermi liquid theory for fermions with short ranged interactions, such as ${ }^{3} \mathrm{He}$, the response function $K$ is described by the solution of a Landau-Boltzmann equation [11. However, for fermions with long ranged interactions [11, the Silin extension of the Landau theory asserts that it is not the function $K$ that is described by the Landau-Boltzmann equation, but rather a response function called $\Pi$. In the case of electrons at zero magnetic field in 2D, for example, $\Pi$ is defined by Eq. 11, but with $c(q)=0$. Eq. 1 separates out the Hartree part of the diverging long ranged interaction such that $\Pi$ gives the quasiparticle response to the sum of the external vector potential and the induced internal vector potential. Within this theory, the Landau-Boltzmann equation describes the dynamics of quasiparticles of mass $m^{*}$ near the Fermi surface [6,7, 10, 11]. The short ranged effective interactions between two quasiparticles whose momenta differ by an angle $\theta$ is described by a function $f(\theta)$. The 'Landau coefficients' are defined as $f_{l}=\frac{1}{2 \pi} \int_{0}^{2 \pi} d \theta f(\theta) e^{i l \theta}$ (by symmetry, $f_{l}=f_{-l}$ ). The parameters $f_{0}$ and $f_{1}$ are fixed by the identities [11]

$$
\frac{d \mu}{d n}=\frac{2 \pi \hbar^{2}}{m^{*}}+f_{0} \quad ; \quad \frac{1}{m_{b}}=\frac{1}{m^{*}}+\frac{f_{1}}{2 \pi \hbar^{2}} .
$$

The second identity is a result of Galilean invariance [11].

Since in the Chern-Simons problem, the interactions are also long ranged, we have similarly made the separation [1] defined in Eq. 目 (with $\left.c(q)=i \Phi_{0} \tilde{\phi} / q\right)$. However, in the limit $m_{b} \rightarrow 0$, further separation should be carried out to remove the diverging magnetization effects. To this end we define a response function $\tilde{\Pi}$ by

$$
\Pi=M \tilde{\Pi} M^{\dagger} .
$$

definition, $\tilde{\Pi}$ relates the transport current of the magnetized quasiparticles to the effective vector potential (including both external and internally induced contributions [10]). It is $\tilde{\Pi}$ which we claim is given by a LandauBoltzmann equation describing the dynamics of quasiparticles with the finite effective mass $m^{*}$.

Recent works attempting to formulate a LandauBoltzmann equation for the Chern-Simons problem [7]:8] pointed out that in Eq. 6, $\frac{d \mu}{d n}$ is taken at fixed $\Delta B$. This yields an $f_{0}$ (as well as an $f_{1}$ ) on the scale of $\mathcal{O}\left(m_{b}^{-1}\right)$ in the limit $m_{b} \rightarrow 0$. We will now show how an $\mathcal{O}\left(m_{b}^{-1}\right)$ value of $f_{0}$ is consistent with our attachment of magnetization. Fixing $\Delta B$ at zero means that when we add a fermion to the system, the external field must also be 
increased by a total of $\tilde{\phi}$ flux quanta. Thus, the magnetic field is linked to the density $n$ via $B=\tilde{\phi} n \Phi_{0}$. The interaction energy between the magnetization $\mathbf{M}=\mu_{b} n$ and the external field is given by $E=\mathbf{M} \cdot \mathbf{B}=\pi \tilde{\phi} \hbar^{2} n^{2} / m_{b}$. Differentiating this with respect to $n$ we obtain a magnetization contribution to the chemical potential $\mu^{\text {mag }}=$ $2 \pi \tilde{\phi} \hbar^{2} n / m_{b}=\hbar \omega_{c}$ such that the magnetization contribution to $f_{0}$ is $\tilde{f}_{0}=d \mu^{\mathrm{mag}} / d n=2 \pi \tilde{\phi} \hbar^{2} / m_{b}$ which is also the inverse compressibility of free electrons of mass $m_{b}$ at constant $\Delta B$. The coefficient $f_{0}$ is written $f_{0}=\tilde{f}_{0}+\delta f_{0}$ where $\tilde{f}_{0}$ is $\mathcal{O}\left(m_{b}^{-1}\right)$ and $\delta f_{0}$ is on the smaller interaction scale. As mentioned in Ref. [7], in the limit $m_{b} \rightarrow 0$, the requirement that the low energy spectrum is independent of $m_{b}$ forces the other interaction coefficients $\left(f_{l}\right.$ for $l \neq 0,1)$ to be on the interaction scale [10].

Since in the limit of $m_{b} \rightarrow 0, \tilde{f}_{0}$ and $f_{1}$ are on the bare mass scale whereas all other coefficients $f_{l}$ (as well as $\delta f_{0}$ ) are on the interaction scale, we will separate out the contributions of these two coefficients by writing [10]

$$
\tilde{\Pi}^{-1}=\left[\tilde{\Pi}^{*}\right]^{-1}+\tilde{\mathcal{F}}_{0}+\mathcal{F}_{1}
$$

where $\tilde{\mathcal{F}}_{0}=\operatorname{diag}\left[\tilde{f}_{0}, 0\right]$. The function $\tilde{\Pi}^{*}$ is to be calculated using a Landau-Boltzmann equation representing quasiparticles with the same effective mass $m^{*}$ and interaction coefficients $f_{l}$ except that $f_{1}$ is artificially set to zero and the magnetic contribution $\tilde{f}_{0}$ is subtracted off of $f_{0}$. The separation of the coefficient $f_{0}$, equivalent to taking $v(q) \rightarrow v(q)+f_{0}$ in Eq. 1, is achieved by noting that $f_{0}$ corresponds to a short ranged density-density interaction. The separation of the nonzero $f_{1}$ coefficient [6, 10] is analogous to that described in Eq. 2 and is derived in Ref. [6]. Having made this separation, we expect that the response $\tilde{\Pi}^{*}(q, \omega)$ is independent of $m_{b}$ in the limit $m_{b} \rightarrow 0$ and is well behaved for all values of $q / m_{b}$.

In Fermi liquid theory, the Landau-Boltzmann equation does not correctly describe the Landau diamagnetic contribution to the transverse static response. Similarly, we suspect that here the function $\tilde{\Pi}_{11}^{*}$ derived from the Landau-Boltzmann equation lacks a term of the form $q^{2} \chi$ where $\chi$ is some appropriate Landau susceptibility which we expect to be on the scale of the interaction strength. As usual, if we fix the ratio $\omega / q$ to be nonzero, and take $q \rightarrow 0$, this diamagnetic term becomes negligible.

Using the identities $\tilde{\mathcal{F}}_{0}=M^{\dagger} \tilde{\mathcal{F}}_{0} M$ and $U+\tilde{\mathcal{F}}_{0}=$ $M^{\dagger-1} U M^{-1}$ (which holds in the limit $m_{b} \rightarrow 0$ ), we find that $\mathrm{M}^{2} \mathrm{RPA}$ is equivalent to approximating $\Pi^{*}$ by $K^{0 *}$, the response of a free Fermi gas of particles of mass $m^{*}$, and calculating the response using Eqns. 1, 0, and 8.

While the $\mathrm{M}^{2} \mathrm{RPA}$ describes well the excitation spectrum of fractionally quantized Hall states $\nu=\frac{p}{2 m p+1}$ for large $p$ at low wavevector $q$, it does not do so at high $q$. In particular, excitations at high $q$ are sensitive to the infrared divergence of the effective mass due to the gauge field fluctuations [7],8] which are neglected in $\mathrm{M}^{2} \mathrm{RPA}$.

To conclude, the transformation Eqns. 1, 2, 3, 8, and 8 do not in themselves involve any approximations, and may be considered simply as a means of defining a new 'irreducible' response function $\tilde{\Pi}^{*}(q, \omega)$. Our claim that $\tilde{\Pi}^{*}$ is well behaved in the limit $m_{b} \rightarrow 0$ we believe to be an exact statement (although we have not proved it rigorously) independent of the approximation used to define the $\mathrm{M}^{2} \mathrm{RPA}$. (For this to be true it does not matter whether we take the moment $\mu_{\mathrm{M}}$ in Eq. 1 to be precisely $\mu_{b}$ or whether we include a correction of order $\mu_{b} m_{b} / m^{*}$.)

The approximation we introduce, the $\mathrm{M}^{2} \mathrm{RPA}$, describes the $\nu=\frac{1}{2 m}$ state as a Fermi liquid of magnetized composite fermions with a finite renormalized effective mass $m^{*}$, an $f_{1}$ parameter dictated by Galilean invariance and an $f_{0}$ parameter originating from the interaction of the magnetization with the magnetic field. All remaining Fermi liquid parameters (which are expected to be on the much smaller interaction scale) are neglected. The $\mathrm{M}^{2} \mathrm{RPA}$ predicts the same $K_{00}$ as the MRPA, but in contrast it yields the correct behavior for $K_{01}, K_{10}$, and $K_{11}$ in the limit $m_{b} \rightarrow 0$ for arbitrary small $q$. In the limit $q \rightarrow 0$, for fixed $m_{b} \neq 0$, the $\mathrm{M}^{2} \mathrm{RPA}$ and MRPA become identical for all components of $K_{\mu \nu}$.

We thank P. A. Lee and D. Orgad for helpful discussions. This research was supported by NSF Grants No. DMR-94-16910 and DMR-95-23361.

[1] B. I. Halperin, P. A. Lee, and N. Read, Phys. Rev. B 47, 7312 (1993); A review of progress on $\nu=\frac{1}{2}$ is given by B. I. Halperin in S. Das Sarma and A. Pinczuk eds. New Perspectives in Quantum Hall Effect (Wiley, in press).

[2] J. K. Jain, Adv. Phys. 41, 105 (1992), and therein.

[3] A. Lopez and E. Fradkin, Phys. Rev. B 445246 (1991); ibid. 47, 7080 (1993);

[4] V. Kalmeyer and S.-C. Zhang, Phys. Rev. B 46, 9889 (1992).

[5] A thorough review of the experimental situation is given by R. L. Willett, to be published in Advances in Physics.

[6] S. H. Simon and B. I. Halperin, Phys. Rev. B 48, 17386 (1993); ibid. 50, 1807 (1994); S. He, S. H. Simon, and B. I. Halperin, ibid. 50, 1823 (1994).

[7] A. Stern and B. I. Halperin, Phys. Rev. B 52, 5890 (1995); to be published in Surf. Sci.

[8] Y. B. Kim, et. al., Phys. Rev. B 50, 17917 (1994); Y. B. Kim, P. A. Lee, and X.-G. Wen, ibid. 5217275 (1995).

[9] The division into $\mathbf{j}_{\text {trans }}$ and $\mathbf{j}_{\text {mag }}$ has some degree of arbitrariness. The definitions used here differ, for example, from those used by Cooper, Halperin, and Ruzin (to be published) in a study of thermoelectric transport in strong magnetic fields which require that $\mathbf{j}_{\text {trans }}=0$ in thermoelectric equilibrium. The definitions in the present paper allow for a nonzero transverse component of $\mathbf{j}_{\text {trans }}$ in equilibrium for an inhomogeneous interacting electron system.

[10] S. H. Simon, A. Stern, and B. I. Halperin, to be published.

[11] D. Pines and P. Nozières, Theory of Quantum Liquids, v. I (Benjamin, New York, 1966); P. Nozières, Theory of Interacting Fermi Systems (Benjamin, New York, 1964). 